\title{
Relative Control of a Virtual Telescope Using GPS and Optical Metrology
}

\author{
Laura Perea * \\ Consejo Superior de Investigaciones Científicas and Institut d'Estudis \\ Espacials de Catalunya, 08034 Barcelona, Spain \\ Jean-Sébastien Ardaens ${ }^{\dagger} \quad$ Simone D’Amico ${ }^{\ddagger}$ \\ Deutsches Zentrum für Luft- und Raumfahrt, 82230 Weßling, Germany \\ Pedro Elosegui $\S$ \\ Consejo Superior de Investigaciones Científicas and Institut d'Estudis \\ Espacials de Catalunya, 08034 Barcelona, Spain
}

\section{Nomenclature}

$$
\begin{array}{ll}
\text { Roman } & \text { Symbols } \\
a & =\text { Semimajor axis } \\
A & =\text { Plant matrix } \\
B & =\text { Deterministic matrix } \\
e & =\text { Eccentricity } \\
e_{c} & =\text { Control error vector } \\
G M & =\text { Gravitational parameter } \\
i & =\text { Inclination } \\
K & =\text { Gain matrix } \\
l & =\text { Reference separation between the occulter and the coronagraph } \\
M & =\text { Mean anomaly } \\
Q & =\text { Control error weighting matrix }
\end{array}
$$

\footnotetext{
${ }^{*}$ Research Assistant, Institute for Space Sciences, CSIC, 08034 Barcelona, Spain; perea@ice.csic.es.

${ }^{\dagger}$ Scientist, Deutsches Zentrum für Luft- und Raumfahrt, 82230 Weßling, Germany; jeansebastien.ardaens@dlr.de.

${ }^{\ddagger}$ Scientist, Deutsches Zentrum für Luft- und Raumfahrt, 82230 Weßling, Germany; simone.damico@dlr.de.

${ }^{\S}$ Scientist, Institute for Space Sciences, CSIC, 08034 Barcelona, Spain; pelosegui@ice.csic.es.
} 


\begin{tabular}{|c|c|}
\hline$R$ & $=$ Control input weighting matrix \\
\hline$r$ & $=$ Position in Earth-Centered Inertial $(\mathrm{ECI})$ \\
\hline$u$ & $=$ Control input \\
\hline$v$ & $=$ Velocity in ECI \\
\hline$x$ & $=$ State vector in ECI \\
\hline \multicolumn{2}{|c|}{ Greek Symbols } \\
\hline$\omega$ & $=$ Argument of perigee \\
\hline$\Omega$ & $=$ Right ascension of ascending node \\
\hline \multicolumn{2}{|c|}{ Subscripts } \\
\hline$c s$ & $=$ The coronagraph satellite \\
\hline cs, ref & $=$ Reference trajectory for the coronagraph \\
\hline $\max$ & $=$ Maximum value \\
\hline os & $=$ The occulter satellite \\
\hline$\oplus$ & $=$ The Earth \\
\hline$\odot$ & $=$ The Sun \\
\hline
\end{tabular}

\section{Introduction}

The benefits of satellite formation flying (FF), whereby a number of individual spacecraft can operate cooperatively to define a single mission, have been widely discussed throughout the space engineering literature. ${ }^{1}$ Some of the remarkable advantages that FF offers over single monolithic satellites are baseline reconfiguration and redundancy, which together can enhance scientific return and lessen the impact of possible technical failure. In most FF missions $^{1,2}$ the trajectories of the satellites are defined by natural orbits and are optimized for long-term stability and control minimization. However, the applications roadmap of FF missions also demands for non-natural, relative trajectories. This is the case of the third Project-for-Onboard-Autonomy (PROBA-3) mission, ${ }^{3}$ which is part of the In-orbit Technology Demonstration Programme of the European Space Agency (ESA).

PROBA-3 was conceived to demonstrate technologies that will be required for autonomous guidance, navigation, and control (GNC) of FF missions of multiple spacecraft. Currently in its preparatory study phase, PROBA-3 will comprise two independent, three-axis stabilized spacecraft flying close together, and will have the ability to control the attitude of both satellites, as well as their relative separation, with high accuracy. Utilizing Global Positioning System (GPS) and laser-based, optical metrology data for accurate navigation and control, as well as cold-gas or electrical thrusters for agile maneuvering, the combined system is expected to achieve mm-level accuracy in relative positioning over satellite separations ranging 
between 25 and $250 \mathrm{~m}^{1}$

The two PROBA-3 satellites will operate as a virtual telescope enabling the study of the Sun's photosphere, with one satellite, the occulter, casting a shadow on the second satellite, the coronagraph, which carries the scientific payload instrument. Scientific operations require that during data collection, the distance between the two elements of this virtual telescope and the alignment of its axis with the Sun's direction remain unchanged, which a control law must guarantee. In [4], the authors presented a GNC system concept for a complete set of PROBA-3 mission phases (i.e., experimental, non-experimental, and deployment). Their control law was designed as a Non-Linear Dynamic Inversion (NDI) with reference dynamics provided by a Proportional-Integral-Derivative (PID) controller. They also used a functional (i.e., software) engineering simulator for algorithm validation. [5] proposed several control laws and carried out a detailed performance assessment. The main control law therein implicitly used Lyapunov's stability theory for the design of a nonlinear expression and its proof of convergence. However, that main control law did not outperform the other simpler algorithms, such as the Linear Quadratic Regulator (LQR) solution, except under very particular conditions.

The LQR technique has been extensively used in FF attitude and orbit control systems, mainly for long-term maintenance. ${ }^{6-8}$ For example, the LQR is used in [9] through a tree digraph representation of the relative along-track displacements for the control of satellite constellations. In [10] a linear pulse control is developed based on a discretization of the dynamics and the LQR technique. More recently, a digital LQR control scheme has been presented in [11] to maintain the separation of the tetrahedron constellation along an elliptical orbit using the linearized Tschauner-Hempel equations of motion. In [5], the authors developed a control scheme to follow a non-natural relative trajectory along a high elliptical orbit (HEO).

This study focuses on the design and validation of a control law for FF acquisition and keeping of a virtual telescope that follows a non-natural relative trajectory, as is the case with the PROBA-3 mission. The control law that is presented in Section II is based on an LQR solution of a time-invariant linear system and, because of its simplicity, could be used for onboard autonomous control of PROBA-3. In this mission, the control system is expected to interact with a navigation system that relies on data from GPS, an optical relative metrology system, or both. Emphasis has thus been given to performance validation of the control law under realistic experimental scenarios using hardware-in-the-loop simulations, as described in Section III. The hardware-in-the-loop ${ }^{12}$ simulations were carried out on the formation flying testbed (FFTB) platform at the German Space Operations Center (GSOC), which includes a GPS navigation algorithm originally developed for the PRISMA FF mission. ${ }^{13} \mathrm{~A}$ simple algorithm for optical navigation was also embedded in the FFTB to simulate PROBA- 
3 experimental conditions. Results from the performance evaluation of the of complete GNC system for a PROBA-3-like configuration are presented in Section IV.

\section{Design of an Autonomous Relative Control System}

The relative control algorithm discussed here involves an autonomous FF mission composed of two spacecraft in a HEO around Earth operating as a virtual telescope for astronomical observations. The control system is therefore suitable for the PROBA-3 mission which, as indicated above, consists of two spacecraft, known as occulter and coronagraph after their respective payload. For such a mission, the astronomical object of interest is the Sun, with which the two-element virtual telescope must be aligned to enable operations during the apogee passage. Based on the mission characteristics of PROBA-3, the occulter is assumed to follow a natural trajectory along a HEO. The coronagraph, in turn, contains an onboard control system, which must ensure that the coronagraph is shadowed by the occulter, is kept at a fixed distance from it, and is aligned with the occulter and the Sun.

Furthermore, due to the significant forces exerted on the telescope at low orbital altitudes, ${ }^{5}$ which will disrupt the FF, the coronagraph is also assumed to perform some collision avoidance maneuvers before the perigee passage and to have all formation controls disabled at those altitudes. After emergence from perigee and once the formation reaches a predetermined altitude, the formation will need to be (re-)acquired from a variable initial error and be kept in FF configuration to within mm-level accuracy for the remainder of the apogee passage. For the design of a continuous control, it is therefore assumed that the coronagraph will carry a low-thrust propulsion system that can operate at high frequency and with mN-level accuracy.

In [5], three control laws were introduced and assessed based on different approximations of the relative dynamics. The main control therein consisted of two terms: the first served to cancel relative accelerations; the second was a time-invariant, linear term. The other two control laws, on the other hand, did not include the first term. Numerical simulations showed that the cancellation of relative accelerations resulted in a significant reduction in relative position error when the formation was close to its reference position and the relative accelerations were large. This result, together with the appealing simplicity of a timeinvariant linear system for autonomous onboard control, justified the selection of the linear control for formation acquisition.

\section{II.A. Formation acquisition relative control}

Building on those results, the linear control function proposed has the form $u=-K e_{c}$, where the gain matrix $K$ is now the solution of an LQR control. To consider such a solution, 
a linear approximation of the control error dynamics is also desired. Such approximation for the optimization of $K$ is possible under the hypothesis that the formation follows a periodic orbit around Earth and that the inter-vehicle separation is small compared to the distance to Earth. ${ }^{5}$ The approximation consists in

$$
\dot{e}_{c}=A e_{c}+B u, \quad A=\left(\begin{array}{cc}
0 & I_{3} \\
-\frac{G M_{\oplus}}{\left\|r_{o s}\right\|^{3}} I_{3} & 0
\end{array}\right), \quad \text { with } \quad B=\left(\begin{array}{c}
0 \\
I_{3}
\end{array}\right)
$$

where $e_{c}=x_{c s}-x_{c s, r e f}$, and $x=(r, v)$ is in an ECI frame.

Note that because the time dependence of $A$ comes through $r_{o s}$, and the $G M_{\oplus} /\left\|r_{o s}\right\|^{3}$ term is small (e.g., less than $10^{-7} s^{-2}$ at an altitude of $17000 \mathrm{~km}$ ), the effect of variations of this term on the LQR solution is not significant (e.g., less than $0.004 \%$ in $2 \mathrm{hr}$ ). Hence, in practice, the term $G M_{\oplus} /\left\|r_{o s}\right\|^{3}$ can be assumed to be constant over time segments of appropriate duration, thus incorporating time-invariance into the dynamics by splitting the orbit in individual arcs. This formulation enables the definition of the control input $u$ as an LQR solution for an invariant system. ${ }^{5,14-16}$

\section{II.B. Formation keeping relative control}

The time-invariant, linear approximation above, though justified for the definition of a control law, may lead to some level of error if such control is applied for sufficiently long periods. The error would have a deleterious effect on the resolution of the virtual telescope and thus the quality of its observations. Therefore, the relative control presented above will be used for formation acquisition, whose accuracy requirements are typically more relaxed than those of formation keeping, and a slight modification will be introduced to improve the accuracy of the latter. The aim here is to reduce the cumulative error as well as the error in relative position and velocity during formation keeping maneuvers. To accomplish this, the state vector is extended to also include the integral of the position, thus $x=\left(\int r d t, r, v\right)$, and define the extended error vector as before, $e_{c}=x_{c s}-x_{c s, r e f}$. The associated dynamic and projection matrices are now

$$
A=\left(\begin{array}{ccc}
0 & I_{3} & 0 \\
0 & 0 & I_{3} \\
0 & -\frac{G M_{\oplus}}{\left\|r_{o s}\right\|^{3}} I_{3} & 0
\end{array}\right), \quad \text { and } \quad B=\left(\begin{array}{c}
0 \\
0 \\
I_{3}
\end{array}\right)
$$

As before, time-invariant systems along consecutive orbit arcs lead to the definition of a new linear control $u=-K e_{c}$, where $K$, in this case, is the solution of the LQR problem for the 
extended system. Note that the additional computational needs associated with calculating the integral of the position error is minor since it can be approximated by the cumulative sum of the errors (from steady-state) times the sampling time.

\section{Validation Using an Experimental Platform}

The validation of the control scheme presented above involved running simulations in the GPS hardware-in-the-loop FFTB developed at GSOC. The testbed was originally designed for the validation of the navigation and control of the flight software of PRISMA, ${ }^{12,13}$ but can be adapted to other real-time embedded GNC systems such as PROBA-3. Since there are substantial differences between PRISMA and PROBA-3, some FFTB upgrades were necessary for simulations involving the latter mission. The following sections describe the most relevant features of the FFTB, the upgrades that were implemented, and the complete set of GNC algorithms embedded in the platform.

\section{III.A. The formation flying testbed}

The GSOC FFTB integrates four units to simulate the different aspects of an FF mission. The spacecraft dynamics and characteristics are emulated by the flight control computer (FCC), which computes the orbit and attitude of each spacecraft using precise force models of the dynamics (see below) and takes into account the effect of the control actions sent by the onboard software. This unit also drives the GPS signal simulator (GSS), a Spirent GSS7700, which simulates the radio frequency signals that are fed to each GPS antenna in the formation. These signals are processed by two single-frequency Phoenix GPS receivers that feed the Onboard Computer (OBC), which, in turn, embeds the complete GNC flight software built from Matlab/Simulink S-functions. Both the FCC and the OBC currently share the same x86 architecture. (See [12] for further details on the platform architecture and models.)

\section{III.B. Upgrades to the FFTB}

Two specific upgrades were necessary to make the FFTB platform suitable for the validation of the control scheme presented for PROBA-3. First, a distinctive feature of PROBA-3 is the shadow that the occulter casts on the coronagraph surface. The relative Solar Radiation Pressure (SRP) and Earth's gravity are the two most important contributions to the relative dynamics between the two spacecraft, ${ }^{5}$ but the former did not describe the peculiar configuration of PROBA-3. The dynamical models of the FCC were therefore modified to account for the effect of the occulter shadow on the coronagraph in the computation of the relative 
SRP. Second, the FFTB metrology system was limited to using only GPS sensors. At high HEO altitudes (where no GPS satellites are in view), an additional metrology system was thus required. As envisaged for PROBA-3, an optical metrology system consisting in optical sensors $^{17}$ were modeled to provide high-accuracy estimates of the inter-vehicle separations. The PROBA-3 optical metrology system is supposed to provide observations at a sampling rate of $10 \mathrm{~Hz}$, with $1 \mathrm{~cm}$ lateral accuracy and 1 arcsec longitudinal accuracy. Note that for the baseline length for Sun observation (i.e., $150 \mathrm{~m}$ ), lateral errors dominate the observations error budget. Table 1 summarizes the main characteristics of the sensors and actuators considered in the final configuration of the FFTB.

Table 1: FFTB sensors and actuators

\begin{tabular}{|l||l|l|}
\hline Signal simulators & Spirent GSS7700 & $\begin{array}{l}\text { Analytical models for optical } \\
\text { data with random noise }\end{array}$ \\
\hline Sensors & $\begin{array}{l}\text { Single-frequency Phoenix GPS } \\
\text { receivers at } 1 \mathrm{~Hz}\end{array}$ & Optical data at $10 \mathrm{~Hz}$ \\
\hline Actuators & \multicolumn{2}{|c|}{ Low-thrust propulsion system } \\
\hline
\end{tabular}

\section{III.C. GNC algorithms embedded in the FFTB}

The navigation system for PROBA-3 relies on two metrology units working in tandem: GPS and the relative optical system. Figure 1 illustrates the architecture of the GPS-based navigation system for PROBA-3, which reproduces the architecture of PRISMA. This system is split into three modules that are integrated in the onboard software of the coronagraph and are run at different rates. First, the GPS interface (GIF) receives GPS messages issued by the Phoenix GPS receivers, at a rate of one message per second, and performs data collection and coarse editing prior to GPS-based orbit determination. Second, the GPS orbit determination (GOD) runs an extended Kalman filter to process group and phase ionospheric correction (GRAPHIC) observables, as well as single-difference carrier-phase measurements, at a rate of once every $30 \mathrm{~s}$. Attitude data from both spacecraft and a history of maneuver data is provided to GOD for orbit determination. GOD performs a numerical orbit propagation that is invoked after the measurement update and provides the GPS orbit prediction (GOP) with orbit coefficients for interpolation for both spacecraft. Last, the GOP module interpolates the orbit coefficients provided by the GOD and supplies the various GNC core functions, and the payload, with continuous position and velocity data of both vehicles at $1 \mathrm{~Hz}$ rate. Due to the different data rates of the GPS-based navigation modules, orbit maneuver data have to be taken into account in both GOD and GOP. In particular, at each GNC step, the GOP must account for maneuvers that were considered by the GOD in the last orbit determination/prediction process. Table 2 describes the force 
models included in the propagation step in the GOD.

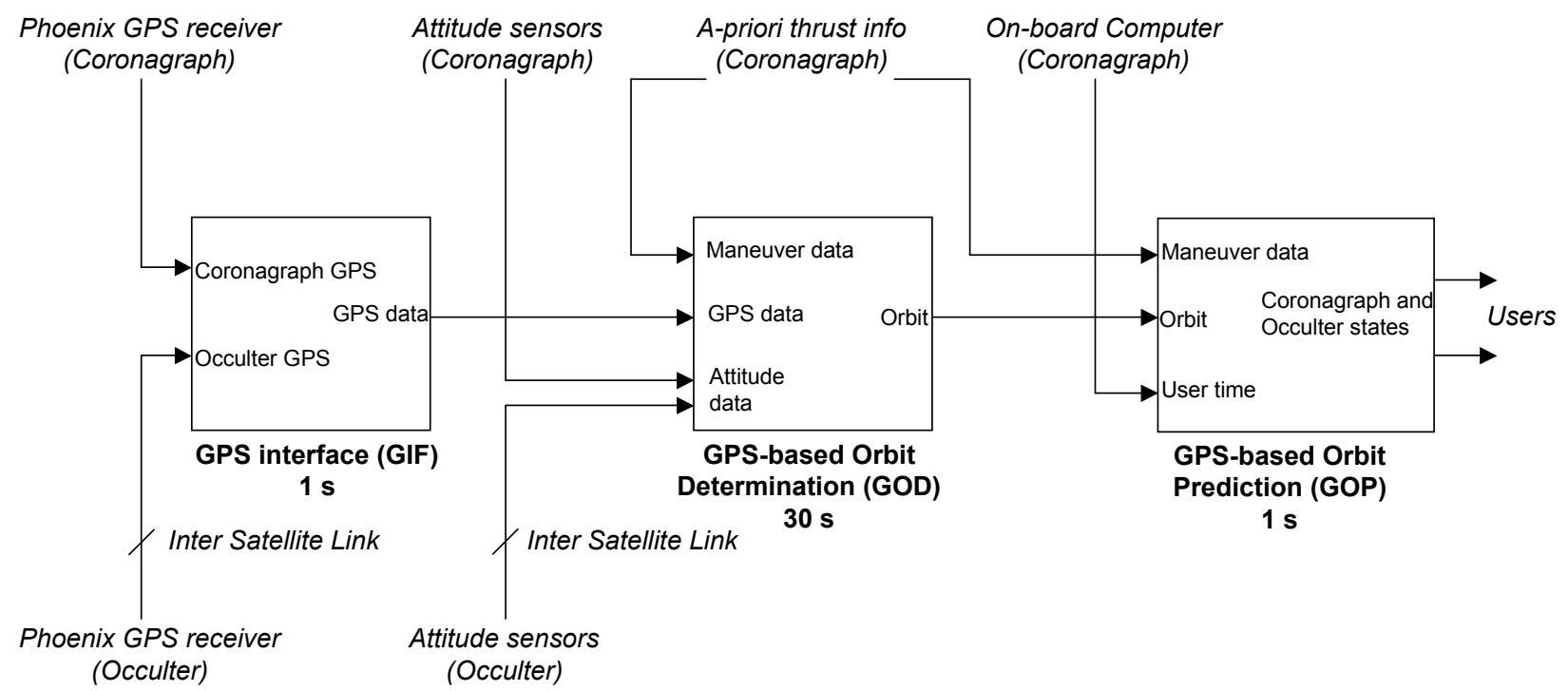

Figure 1: Proposed architecture of the GPS-based navigation system for PROBA-3.

The architecture of the optical-based navigation system is quite simple. This system comprises two components, determination and prediction, which run once every $6 \mathrm{~s}$ and $1 \mathrm{~s}$, respectively. In this case, the determination unit has been designed as a Least Squares Filter (LSQ) that combines cumulated optical observations, collected at $10 \mathrm{~Hz}$, over the last $6 \mathrm{~s}$. The optical navigation system is used at high altitudes, where forces such as Earth's oblateness and the atmospheric drag are negligible, and the propagator is based on a simple Keplerian model. The inclusion of the SRP in this propagation was deemed unnecessary since the maximum integration duration is a few seconds.

Eqn (3) defines the guidance system implemented for the occulter. For this computation, the system uses the relative position and velocity of the coronagraph on the reference trajectory. These are calculated using a low-precision ephemeris of the Sun and the estimated position of the occulter which, at high altitudes, is a propagation of the last estimate obtained from the GPS data.

$$
x_{c s, r e f}=x_{o s}+l\left(\frac{x_{o s}-x_{\odot}}{\left\|x_{o s}-x_{\odot}\right\|}\right)
$$

\section{III.D. Scenario}

The reference orbit for PROBA-3 is a HEO to avoid perturbations of the relative dynamics during the apogee passage, when the virtual telescope is collecting scientific data. The Keplerian parameters of this orbit are $(a, e, i, \Omega, \omega, M)=(42241 \mathrm{~km}, 0.778,0.6981 \mathrm{rad}$, 
Table 2: Force models included in the FCC and the navigation algorithms of PROBA-3

\begin{tabular}{|c|c|c|}
\hline & FCC & PROBA-3 \\
\hline Earth gravity & $\begin{array}{l}\text { GRACE Earth Gravity Model } \\
(\text { GGM01S })^{2}\end{array}$ & $\begin{array}{l}\text { Gravity expansion in spherical } \\
\text { harmonics up to order and degree } \\
20^{18}\end{array}$ \\
\hline $\begin{array}{l}\text { Solar perturba- } \\
\text { tions }\end{array}$ & $\begin{array}{l}\text { Analytical ephemeris based on a } \\
\text { modification of Newcomb's Ta- } \\
\text { bles }^{19-21}\end{array}$ & Low-precision solar coordinates ${ }^{18}$ \\
\hline $\begin{array}{l}\text { Lunar perturba- } \\
\text { tions }\end{array}$ & $\begin{array}{l}\text { Analytical ephemeris based on a } \\
\text { modification of the Improved Lu- } \\
\text { nar Ephemeris }{ }^{21-23}\end{array}$ & Low-precision lunar coordinates ${ }^{18}$ \\
\hline $\begin{array}{l}\text { Atmospheric } \\
\text { drag }\end{array}$ & $\begin{array}{l}\text { Gill-Jacchia flux atmospheric } \\
\text { density model }^{24}\end{array}$ & $\begin{array}{l}\text { Harris-Priester atmospheric den- } \\
\text { sity model }{ }^{18}\end{array}$ \\
\hline SRP & $\begin{array}{l}\text { Cannonball model for the Earth } \\
\text { shadow }^{18} \text { and a grid-based conical } \\
\text { model for the occulter shadow }\end{array}$ & $\begin{array}{l}\text { Cannonball model for the Earth } \\
\text { shadow. }{ }^{18} \text { Effect of the occulter } \\
\text { shadow not included }\end{array}$ \\
\hline Tidal effects & IERS model $^{25}$ & Not included \\
\hline $\begin{array}{l}\text { Relativistic } \\
\text { effects }\end{array}$ & Included $^{18}$ & Not included \\
\hline Empirical forces & Not included & Included $^{18}$ \\
\hline
\end{tabular}

$0 \mathrm{rad}, 0 \mathrm{rad}, 1.02 \mathrm{rad}$ ), which is a 1-day orbit. During Sun's corona observations, both spacecraft should be aligned with the Sun, and they should preserve a baseline distance of $150 \mathrm{~m}$ to operate as a virtual telescope.

The various GNC modes are summarized in Table 3. The GPS component of the navigation system requires simultaneous observations from a minimum of four satellites. In the simulations, this requirement can be fulfilled between the perigee and orbital altitudes slightly higher than $17000 \mathrm{~km}$. Therefore, the relative navigation system has been designed to switch from GPS data to optical metrology at that altitude. The control system is enabled when the two spacecraft reach an altitude of $11000 \mathrm{~km}$. (The spacecraft were in free-flying mode before reaching that altitude.) First, the control starts as formation acquisition, which lasts until an altitude of $19000 \mathrm{~km}$. Then the control switches to formation keeping, and is coupled with a navigation based on the optical metrology system.

Table 3: GNC modes of operation

\begin{tabular}{|l|cccc|}
\hline Altitude (in km) & $<11000$ & $11000-17000$ & $17000-19000$ & $>19000$ \\
Time (in orbit-units) & $<0.0047$ & $0.0047-0.0240$ & $0.0240-0.030$ & $0.030-0.960$ \\
\hline Metrology & GPS & GPS & optical & optical \\
Control & free flying & acquisition & acquisition & keeping \\
\hline
\end{tabular}


The optimum solution of the LQR depends on the definition of a cost function $J$, which depends on two weighting matrices, $Q$, for the control error and, $R$, for the control input. ${ }^{14,15}$ These matrices were defined in the simulations according to the maximum size technique, i.e., defining a maximum threshold for the error in the integral, position, and velocity $\left(i_{\max }\right.$, $\left.x_{\max }, v_{\max }\right)$, and for the control input $u_{\max }$. The weighting matrices are then defined as,

$$
Q=\left(\begin{array}{cc}
x_{\max }^{-2} I_{3} & 0 \\
0 & v_{\max }^{-2} I_{3}
\end{array}\right) \quad \text { or } \quad Q=\left(\begin{array}{ccc}
i_{\max }^{-2} I_{3} & 0 & 0 \\
0 & x_{\max }^{-2} I_{3} & 0 \\
0 & 0 & v_{\max }^{-2} I_{3}
\end{array}\right)
$$

for formation acquisition and keeping, respectively, and $R=u_{\max }^{-2} I_{3}$. The selection of optimum values for $i_{\max }, x_{\max }, v_{\max }$, and $u_{\max }$ is usually a critical aspect, ${ }^{5}$ and obviously depends on the control mode. In this case, the set of optimum values were found to be $\left(x_{\max }, v_{\max }, u_{\max }\right)=\left(1,10^{-2}, 10^{-4}\right)$ for formation acquisition, and $\left(i_{\max }, x_{\max }, v_{\max }, u_{\max }\right)=$ $\left(0.1,10^{-3}, 10^{-5}, 10^{-6}\right)$ for formation keeping.

As previously mentioned, the Keplerian orbit is split into a number of non-overlapping arc segments for a time-invariant approximation of the dynamics in the computation of the feedback matrix $K$. Choosing arc segments of 2-h duration results in LQR solutions that vary less than $0.004 \%$ during that time, thus, keeping errors within bounds. Furthermore, the storage capacity of the gain matrices for 2-h updates is also negligible.

The physical characteristics of the spacecraft used in the simulations are summarized in Table 4. For lack of better definition, it is assumed that the Sun-facing surface of the satellites are discs of radius $R_{o s}$ and $R_{c s}$, respectively. The thrusters are assumed to reach $0.04 \mathrm{~N}$ before saturation, and have a thrust resolution of $10^{-6} \mathrm{~N} .^{17}$

Table 4: Physical characteristics of the PROBA-3 spacecraft

\begin{tabular}{|l||cccc|}
\hline & Mass $(\mathrm{kg})$ & Radius $(\mathrm{m})$ & Area $\left(\mathrm{m}^{2}\right)$ & Reflectivity coefficient \\
\hline Occulter & 200 & 1 & 3.1416 & 1.3 \\
Coronagraph & 200 & 0.75 & 1.7671 & 1.3 \\
\hline
\end{tabular}

The FFTB also allows to account for several small transmission and computation delays, which have been fixed in the simulations to values similar to those of PRISMA. Specifically, the values used are $1 \mathrm{~s}$ for data transmission and observer computation delays, $1 \mathrm{~s}$ for control computation and transmission delay, and $0.5 \mathrm{~s}$ for propulsion delay. 


\section{Results and discussion}

Shown in Fig. 2 are the errors of the relative navigation estimates. The large initial error in relative navigation arises from the FFTB, which takes $\sim 1$ min to have all its units activated from a cold start, and, more importantly, from the GPS navigation system, which typically needs $\sim 20 \mathrm{~min}$ for signal acquisition and filter convergence. Furthermore, the control system is enabled at $\sim 0.005$ orbit-time units (see Table 3 ), i.e., before the filter has reached steady-state. In nominal conditions, when no maneuvers are performed before the filter converges, the relative GPS navigation system is expected to reach cm-level accuracy. Despite the initial estimation error caused by an anticipated initiation of maneuvers, and up to $\sim 0.013$ orbit-time units, the navigation error remains at the few-decimeter level (Fig. $2 \mathrm{a}$ ). The navigation filter is able to perform a measurement update at $\sim 0.0185$ orbits-time units. Between that epoch and 0.024 orbits-time units, the quality of the satellite geometry significantly reduces, and the (Kalman) navigation filter responds by implementing only the time update step. A steady increase in navigation error ensues from only propagating the orbit, as one would expect.

The GPS observations are used through $\sim 0.024$ orbit-time units, when the altitude of the formation reaches $17000 \mathrm{~km}$, (see Table 3). By that time, the inter-satellite optical beam has already entered the field of view of the optical sensor, and the navigation system switches from using GPS to using optical data. This result in an immediate improvement in the navigation error budget (Fig. 2a). Since the optical metrology system is supposed to be active in background mode some time before the switch occurs, the filter has already converged at the time of the switch. The position error from that time and for the rest of the orbit is reduced to $0.3 \mathrm{~cm}$, in root-mean-square (RMS) sense (Fig. 2b), and to $0.035 \mathrm{~cm} / \mathrm{s}$ in velocity (not shown). The error budget of relative navigation is summarized in Table 5.

Table 5: Figures of merit of the relative navigation error, in $\mathrm{cm}$, after $20 \mathrm{~min}$ of system activation.

\begin{tabular}{|l|ccc|}
\hline & max & RMS & mean \\
\hline GPS & 22.0 & 16.3 & 15.9 \\
Optical & 1.3 & 0.3 & 0.2 \\
\hline
\end{tabular}

The guidance system needs the positions of the Sun and the occulter to compute the unitary vector in the direction of the occulter shadow (see eq. (3)). As described earlier, the guidance system uses low-precision Sun coordinates and the propagation of the last estimate of the occulter position in an ECI frame that was obtained using GPS data. Although absolute errors in these estimates may be large by the end of the apogee passage, the error of the unitary shadow vector on the reference position of the coronagraph is equal or smaller 

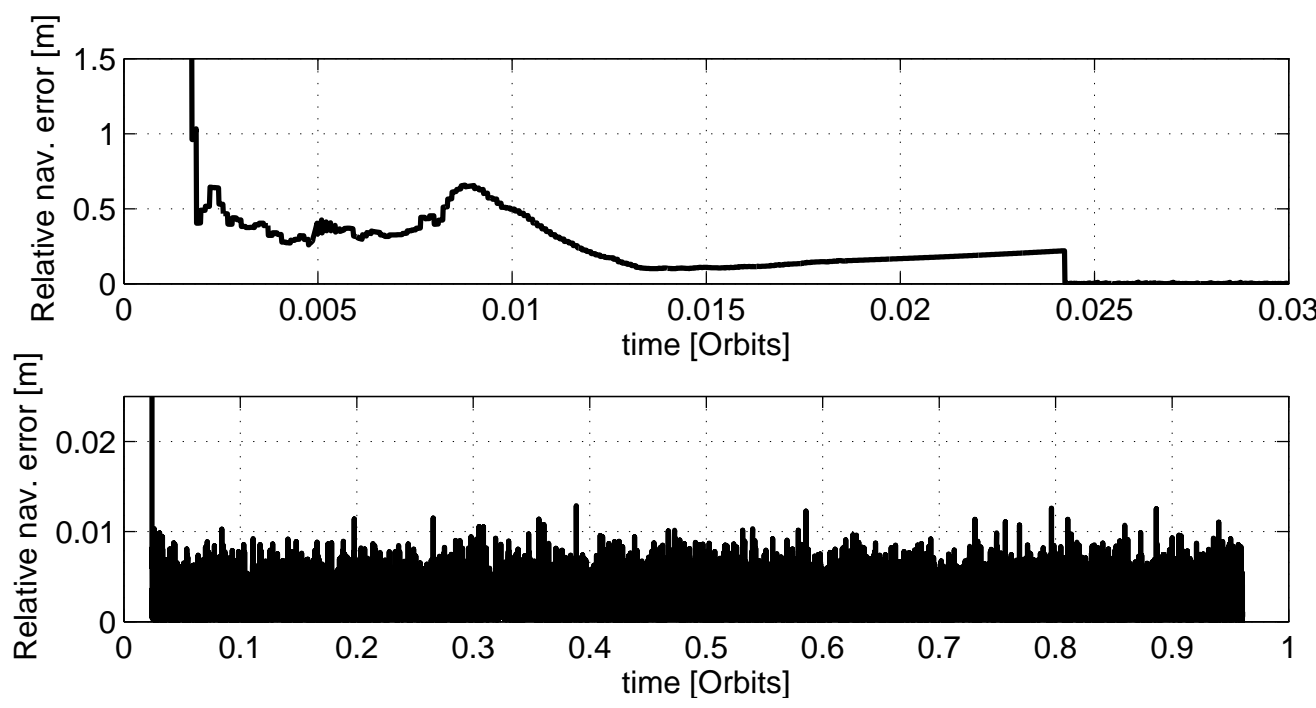

Figure 2: Relative navigation error of PROBA-3. The navigation system uses GPS observations during the perigee passage, i.e., from the beginning of the simulation through $\sim 0.024$ orbit-time units (or an equivalent orbital altitude of $17000 \mathrm{~km}$ ), and the optical metrology system thereafter. (Top) Error during the initial navigation stages, when GPS data is used. (Bottom) Error for the entire orbit. Note the scale changes.

than $0.06 \mathrm{~cm}$ throughout the entire orbit, and $0.03 \mathrm{~cm}$ in RMS sense, thus negligible.

Current mission specifications call for collision avoidance maneuvers to be executed right before the perigee passage while the spacecraft are in free-flying mode. The position error of the coronagraph relative to its reference position at the end of this passage can vary depending on those maneuvers. In the simulations, it is assumed that the perigee passage ends at $\sim 0.005$ orbit-time units (or equivalent altitude of $11000 \mathrm{~km}$ ) and that the control error has grown to $8 \mathrm{~m}$ by that time (Fig. 3), which is a reasonable level of error. Upon enabling the control (at $\sim 0.005$ orbit-time units) in formation acquisition mode, the error decreases quickly, down to $0.47 \mathrm{~m}$ by the end of the GPS relative navigation phase (at $\sim 0.024$ orbit-time units). When the metrology system then switches to using optical observations, the control error continues to decrease, down to $0.21 \mathrm{~m}$ by the end of the control phase in formation acquisition mode (i.e., by $\sim 0.03$ orbit-time units, or $19000-\mathrm{km}$ altitude). At that point, the control switches to formation keeping mode, and the concomitant reduction in control error is very significant, reaching cm level in less than 0.01 orbit-time units (see also Fig. 4). This overall error reduction represents an improvement in control performance of $\sim 2000 \%$, which largely results from the extension of the control law to include a position-integral term.

Except for the initial control action (i.e., at $\sim 0.005$ orbit-time units), the magnitude of the thrusting necessary to achieve this control error is at the level of a few tens of $\mathrm{mN}$, thus within the capabilities of PROBA-3 thrusters. As representative statistical figures, the largest, RMS, and mean value of the control error during formation keeping are 2.48, 0.63, 
and $0.05 \mathrm{~cm}$, respectively. As for the magnitude of the control action, the same statistics are 47,10 , and $8 \mathrm{mN}$.

The control action during the formation-keeping phase contains high-frequency variations in all three components (Fig. 4). (The low-frequency variation in the $\mathrm{x}$ and $\mathrm{y}$ components results from the change in relative orientation of the two spacecraft, which is required to keep the telescope pointing in the direction of the Sun as the spacecraft orbit around the Earth.) Simulations revealed that the high-frequency oscillations arise from errors in relative velocity estimates (not shown), and that the oscillations vanished in the absence of velocity errors. This result implies that further refinements in control error could be gained by reducing the cost of the control by improving the estimates of relative velocity.

Errors in attitude determination could have an effect on the relative orbit control system. However, attitude determination and control of PROBA-3 is expected to achieve arcsec-level accuracy. ${ }^{17}$ Therefore, these errors are not expected to have a perceptible impact on orbit determination and control, and have not been considered in the simulations.

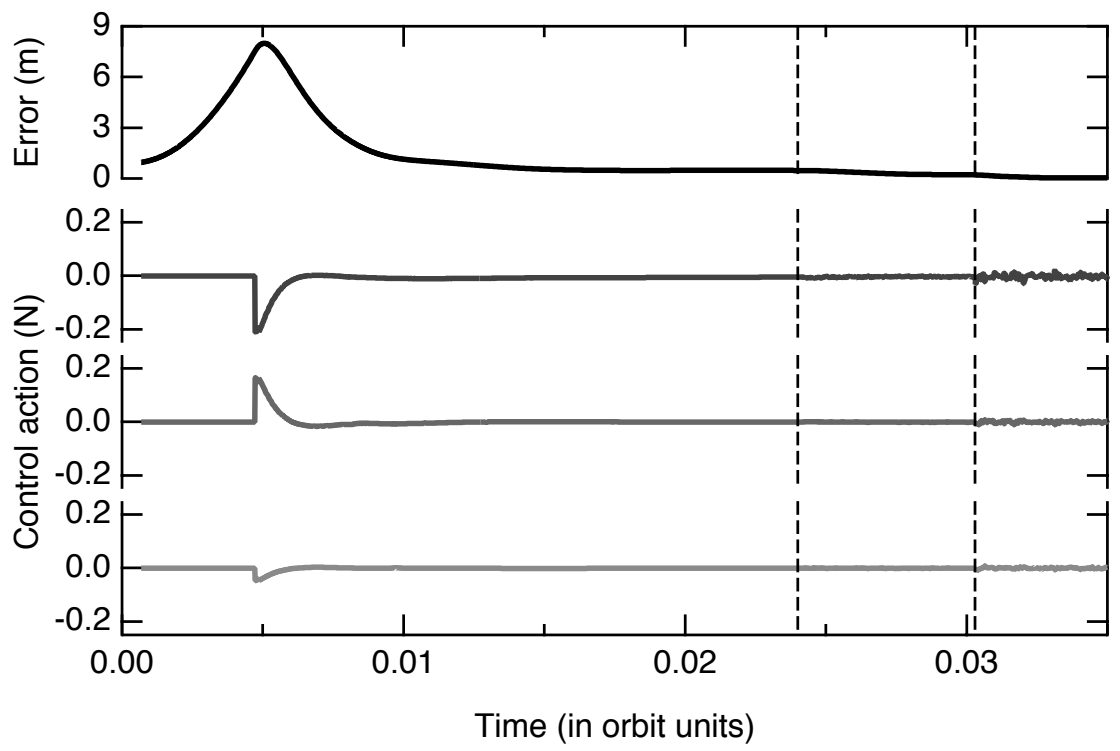

Figure 3: Control figures during formation acquisition maneuvers. (Top) Control error and (second-from-top to last) control input in $\mathrm{x}, \mathrm{y}$, and z components in ECI. Dashed lines mark, at $\sim 0.024$ orbit-time units, the switch of metrology system from GPS to optical data and, at $\sim 0.03$ orbit-time units, the switch of control mode from formation acquisition to formation keeping (see Table 3).

\section{Conclusions}

The guidance, navigation, and control algorithms presented and validated here have been able to acquire the two-element telescope formation of the PROBA-3 mission from an 


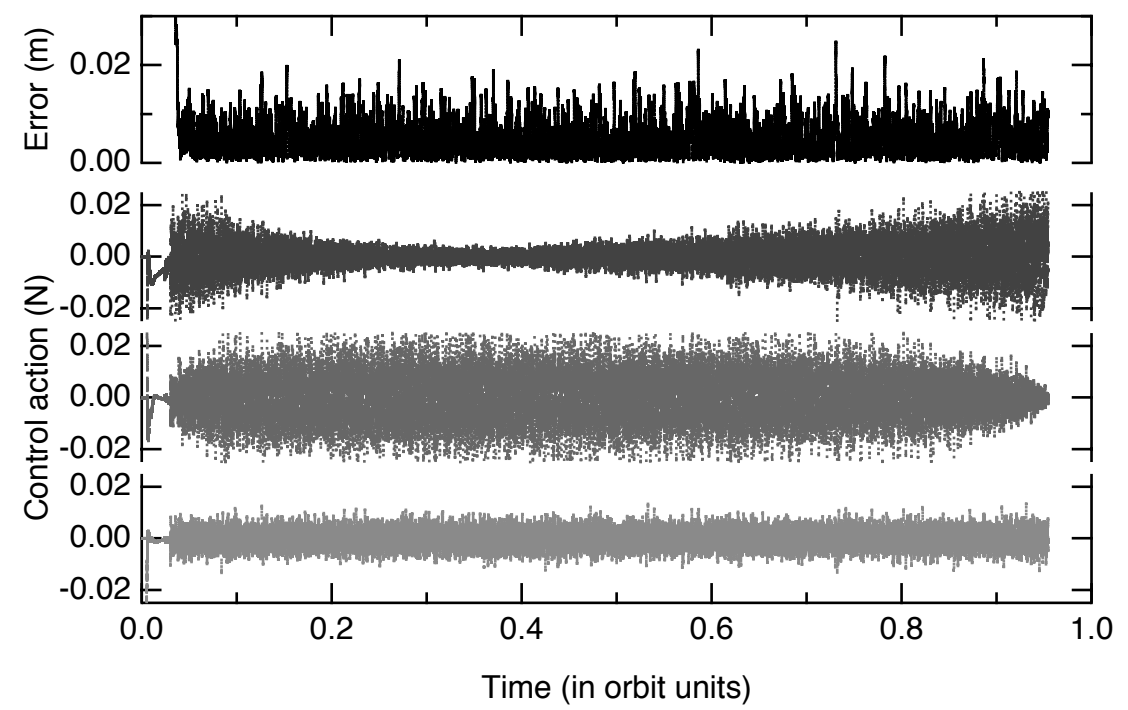

Figure 4: Same as Fig. 3 but here for formation keeping maneuvers.

a priori control error of $8 \mathrm{~m}$. The control error becomes sub-cm (0.6-cm root-mean-square (RMS)) for more than $90 \%$ of its high elliptical orbit. This error level relies on the use of GPS observations for coarse navigation and of an optical system for fine navigation. The navigation error of the latter is $\sim 0.3 \mathrm{~cm}$ RMS. The control presented, a Linear Quadratic Regulator solution of a linear control algorithm, can be used for both formation acquisition and formation keeping. The inclusion of a simple position-integral term in the formation keeping version results in an error reduction of $\sim 2000 \%$ relative to the formation acquisition version.

More generally, this study demonstrates that a simple control design coupled to an accurate navigation system can be used to fulfill stringent formation acquisition and formation keeping requirements. Although the study focuses on the experimental phase of PROBA3 , the present strategy could be easily adapted to other formations in a planetary orbit environment that have to follow non-natural relative trajectories.

\section{Acknowledgments}

Special thanks to Oliver Montenbruck for his guidance and advice. L. P. would also like to express her gratitude to him for his warm welcome, and that of his family, during her stays at the Deutsches Zentrum für Luft- und Raumfahrt (DLR). Editorial assistance from Dee Jay Foster is appreciated. This study was supported by Spanish Ministry of Education and Science (MEC) fellowship BES-2005-8607 (LP) and Ministry of Science and Innovation (MICINN) grant ESP2007-62680. 


\section{References}

${ }^{1}$ D'Amico, S., Ardaens, J.-S., De Florio, S., and Montenbruck, O., "Autonomous Formation Flying TANDEM-X, PRISMA and Beyond," Proceedings of the 5th International Workshop on Satellite Constellations $\& 3$ Formation Flying, Evpatoria, Crimea, July 2008, pp. 1-15.

${ }^{2}$ Tapley, B., Bettadpur, S., Watkins, M., and Reigber, C., "The Gravity Recovery and Climate Experiment: Mission Overview and Early Results," Geophysical Research Letters, Vol. 31, 2004, pp. 1-6. doi:10.1029/2004GL019920

${ }^{3}$ Wishart, A., Teston, F., Kemble, S., Grocott, S., Davies, A., Warren, C., and Geelen, K., "The PROBA-3 formation flying technology demonstration mission," Proceedings of the 58th International Astronautical Congress, Hyderabad, India, Sept. 2007, pp. 1-10.

${ }^{4}$ Di Sotto, E., Peñín, L. F., Bastante, J. C., Marcos, A., and Branco, J., "FF analysis and GNC concept for a FF mission in highly eccentric orbit," Proceedings of the 3rd International Symposium on Formation Flying, Missions, and Technologies, Noordwijk, The Netherlands, June 2008, pp. 1-9.

${ }^{5}$ Perea, L., D'Amico, S., and Elosegui, P., "Relative Formation Flying Dynamics and Control of a TwoElement Virtual Telescope on a HEO," Proceedings of the 21st International Symposium on Space Flight Dynamics (ISSFD), edited by CNES, Sept. 2009, pp. 1-15.

${ }^{6}$ Vassar, R. H., and Sherwood, R. B., "Formationkeeping for a Pair of Satellite in a Circular Orbit," Journal of Guidance, Control, and Dynamics, Vol. 8, March 1985, pp. 235-242. doi: 10.2514/3.19965

${ }^{7}$ Leonard, C. L., Hollister, W. M., and Bergmann, E. V., "Orbital Formationkeeping with Differential Drag," Journal of Guidance, Control, and Dynamics, Vol. 12, Jan. 1989, pp. 108-113. doi: 10.2514/3.20374

${ }^{8}$ Redding, D. C., Adams, N., and Kubiak, E. T., "Linear-Quadratic Stationkeeping for the STS Orbiter," Journal of Guidance, Control, and Dynamics, Vol. 12, March 1989, pp. 248-255. doi: 10.2514/3.20398

${ }^{9}$ Ulybyshev, Y., "Long-Term Formation Keeping of Satellite Constellation Using Linear-Quadratic Controller," Journal of Guidance, Control, and Dynamics, Vol. 21, Jan. 1998, pp. 109-115. doi: 10.2514/2.4204

${ }^{10}$ Kapila, V., Sparks, A. G., Buffington, J. M., and Yan, Q., "Spacecraft Formation Flying: Dynamics and Control," Journal of Guidance, Control, and Dynamics, Vol. 23, May 2000, pp. 561-564. doi: $10.2514 / 2.4567$

${ }^{11}$ Capó-Lugo, P. A., and Bainum, P. M., "Digital LQR control scheme to maintain the separation distance of the NASA benchmark tetrahedron constellation," Acta Astronautica, Vol. 65, 2009, pp. 1058-1067.

${ }^{12}$ Ardaens, J.-S., and D'Amico, S., "Formation Flying Testbed," Deutsches Zentrum für Luft- und Raumfahrt, Tech. Rep. TN 09-01, Oberpfaffenhofen, Germany, Feb. 2009.

${ }^{13}$ D'Amico, S., Gill, E., Garcia, M., and Montenbruck, O., "GPS-Based Real-Time Navigation for the PRISMA Formation Flying Mission," Proceedings of the Satellite Navigation User Equipment Technologies, Noordwijk, Netherlands, Dec. 2006, pp. 1-8.

${ }^{14}$ Kwakernaak, H., and Raphael, S., "Optimal Linear State Feedback Control Systems," Linear Optimal Control Systems, Wiley-Interscience, New York, 1972, pp. 193-327.

${ }^{15}$ Friedland, B., "Linear, Quadratic Optimum Control," Control System Design: An Introduction to State-Space Methods, edited by S. W. Director, McGraw-Hill Higher Education, 1st ed., Jan. 1985, pp. $337-377$.

${ }^{16}$ Sontag, E. D., "Optimality: Value Function," Mathematical Control Theory. Deterministic Finite Dimensional Systems, Texts in Applied Mathematics, Springer-Verlag, 1990, pp. 347-396. 
${ }^{17}$ PROBA-3 phase A team, "PROBA-3 phase A study executive summary report," EADS Astrium, Tech. Rep. PROBA3-ASU-RPT-14, issue 01, Dec. 2007.

${ }^{18}$ Montenbruck, O., and Gill, E., "Force Model," Satellite Orbits. Models, methods and applications, Springer, Berlin, 3rd ed., 2005, pp. 53-116.

${ }^{19}$ Newcomb, S., "Tables of the motion of the earth on its axis and around the sun," Astronomical paper of the American Ephemeris, Vol. 6, 1898, pp. 1-29.

${ }^{20}$ Francou, G., Bergeal, L., Chapront, J., and Morando, B., "Nouvelles ephemerides du Soleil, de la Lune et des planetes," Astronomy and Astrophysics, Vol. 128, No. 1, 1983, pp. 124-139.

${ }^{21}$ Bretagnon, P., and Francou, G., "Planetary theories in rectangular and spherical variables - VSOP 87 solutions," Astronomy and Astrophysics, Vol. 202, Aug. 1988, pp. 309-315.

${ }^{22}$ Nautical Almanac Office, Washington, US, Improved Lunar Ephemeris 1952-1959, 1954.

${ }^{23}$ Chapront-Touze, M., and Chapront, J., "A semi-analytical lunar ephemeris adequate for historical times," Astronomy and Astrophysics, Vol. 190, 1988, pp. 342-352.

${ }^{24}$ Gill, E., Smooth Bi-Polynominal Interpolation of Jacchia 1971 Atmospheric Densities For Efficient Satellite Drag Computation, Deutschen Zentrum für Luft- und Raumfahrt, Oberpfaffenhofen, Germany, 1996.

${ }^{25}$ McCarthy, D., and Petit, G., "IERS Conventions (2003)," Observatoire de Paris, Tech. Rep. 32, 2003. 